\title{
Built-in Self-Diagnosis Targeting Arbitrary Defects with Partial Pseudo-Exhaustive Test
}

\author{
Alejandro Cook ${ }^{1}$, Sybille Hellebrand ${ }^{2}$, Michael E. Imhof ${ }^{1}$, Abdullah Mumtaz ${ }^{1}$, Hans-Joachim Wunderlich ${ }^{1}$ \\ ${ }^{1}$ Institute of Computer Architecture and Computer Engineering, University of Stuttgart, Germany \\ E-mail: \{cook, imhof, mumtazah\}@iti.uni-stuttgart.de,wu@informatik.uni-stuttgart.de \\ ${ }^{2}$ Institute of Electrical Engineering and Information Technology, University of Paderborn, Germany \\ E-mail: sybille.hellebrand@uni-paderborn.de
}

\begin{abstract}
Pseudo-exhaustive test completely verifies all output functions of a combinational circuit, which provides a high coverage of non-target faults and allows an efficient on-chip implementation. To avoid long test times caused by large output cones, partial pseudo-exhaustive test (P-PET) has been proposed recently. Here only cones with a limited number of inputs are tested exhaustively, and the remaining faults are targeted with deterministic patterns. Using P-PET patterns for built-in diagnosis, however, is challenging because of the large amount of associated response data. This paper presents a built-in diagnosis scheme which only relies on sparsely distributed data in the response sequence, but still preserves the benefits of P-PET.
\end{abstract}

Index Terms-Built-in Self-Test, Pseudo-Exhaustive Test, Built-in Self-Diagnosis

\section{INTRODUCTION}

Pseudo-exhaustive testing (PET) was proposed in the 1980 's to guarantee high defect coverage by a simple test generation process also suitable for on-chip implementation [2][8]. Given a circuit with inputs $I$ and outputs $O$, for each output $o \in O$ the set $I(o) \subset I$ contains all inputs the output $o$ depends on. A pseudo-exhaustive test applies all possible input combinations for each input set $I(o)$. Serially exercising all input sets requires at most $|O| \cdot 2^{w}$ test patterns, where $w$ is the cardinality of the largest input set. Thus, a PET is only feasible, if $w$ does not exceed a given limit, e.g. $w=24$.

Meanwhile PET has regained attention, because its high defect coverage independent of specific fault models is particularly attractive in the presence of new defect mechanisms in nanoscale technologies. Furthermore, today's high speed circuits are characterized by limited circuit depths and, consequently, by smaller input sets $I(o)$. Recently, partial pseudo-exhaustive test (P-PET) has been proposed as a solution for arbitrary circuits [9]. Here, exhaustive patterns are applied only to inputs sets of limited size $|I(o)| \leq b$. This way large parts of the circuit can be exhaustively covered,

This work has been performed within the project RealTest sponsored by the German National Science Foundation (DFG-grants HE 1686/3-2 and WU 245/5-2). and for the remaining parts, the generated patterns provide high quality random patterns. Compared to a mixed-mode BIST using pseudo-random patterns, a P-PET complemented with deterministic patterns can provide higher defect coverage with less test data storage.

To exploit the benefits of P-PET also for yield ramp-up and in-field repair, it must be combined with efficient techniques for built-in self-diagnosis (BISD). Built-in diagnosis has been in the focus of research for many years, but most of the approaches either require multiple test runs or are not compatible with a standard scan architecture. The windowbased diagnosis in [4][5] is compatible with STUMPS and supports a fully autonomous BISD in a single test run. Nevertheless, applying this scheme to long test sequences would result in very high storage requirements for the response data. BISD for LBIST and for mixed-mode BIST with long pseudo-random sequences needs additional strategies for reducing the response data [1][6]. As the sequences for P-PET are typically much longer than the pseudo-random sequences in LBIST or mixed-mode BIST, it is even more challenging to limit the storage requirements for response data. This paper shows how the diagnosis approach in [5] can be adapted to efficiently deal with P-PET sequences while maintaining the high defect coverage.

\section{ARCHITECTURE}

As shown in Figure 1 the P-PET architecture proposed in [9] is combined with the window-based diagnosis of [4][5]. To generate a P-PET sequence for a given bound $b$, the multiple-polynomial LFSR (MP-LFSR) switches between several polynomials stored on chip, each one exercising a subset of $I_{b}=\{I(o)|| I(o) \mid \leq b\}$, such that overall each input set in $I_{b}$ receives all possible input combinations. The polynomials are pre-computed relying on the check criterion described in [2]. Deterministic patterns are added to cover those faults, which are not detected by the P-PET sequence. During test, the MP-LFSR is used as a decompressor to regenerate the patterns from the respective seeds stored in the seed memory. 


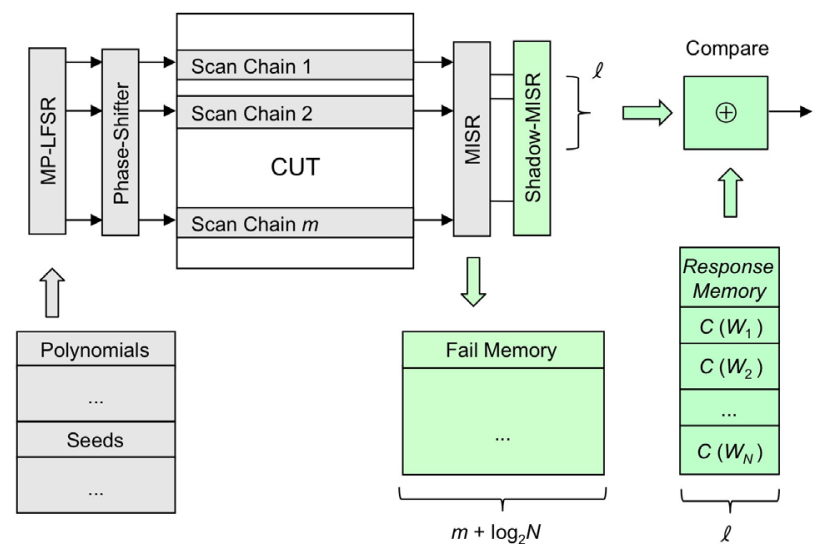

Figure 1. Figure 1: Architecture for BISD with P-PET

For diagnosis the test is partitioned into windows, i.e. short contiguous test sequences. Each window is characterized by a single cumulative signature, which is copied to a shadow-MISR at the end of the window. While the test continues normally with the next window, the shadow MISR runs in autonomous mode as long as the first pattern is applied. This way fault effects are distributed over the MISR randomly, and it is sufficient to observe only $\ell$ bits of the shadow-MISR [5]. The observed bits are compared to the respective reference data stored in the response memory. If a mismatch is detected, the complete signature is stored in the fail memory together with the index of the test window.

\section{DiAgnOSIS}

The window-based diagnosis proposed in [4][5] is based on the conditional stuck-at fault model and computes the fault location directly from the faulty signature. While other schemes for "direct diagnosis" analyze signatures for single patterns [3], here signatures correspond to windows in the test sequence. For each candidate fault location $v$, the circuit responses are determined when a stuck-at-zero or stuck-atone fault at $v$ is activated by a single pattern in the window. From this information a system of linear equations is built, which has a solution, only if the fault location $v$ explains the faulty behavior. To support a unique solution, the dimensions of the system of equations must be properly set. As shown in [4][5], the number of patterns in a window should be less than or equal to the number of bits in the MISR signature. A straightforward application of the scheme to long P-PET sequences would therefore result in an extremely large response memory.

To reduce the response data and keep the high defect coverage, the P-PET sequence is partitioned into "strong" and "weak" diagnostic windows similarly as in [6]. "Strong" windows fulfill the length restrictions of [4][5] and are analyzed with the corresponding approach. "Weak" windows can contain more patterns and are treated with a less expensive diagnostic procedure.
For partitioning the P-PET sequence $T_{P-P E T}$ into strong and weak windows, it is first fault-simulated against a given set of target faults $F$. Whenever a fault is detected for the first time, the respective pattern is stored in the set of candidate essential patterns $E\left(T_{P-P E T}\right)$. Subsequently, ATPGpatterns $T_{d e t}$ are generated for the undetected hard faults. Then the candidate essential patterns and the deterministic patterns $E\left(T_{P-P E T}\right) \cup T_{\text {det }}$ are fault simulated in reverse order, and unnecessary patterns are dropped. This way a reduced set of deterministic patterns $T_{\operatorname{det}}^{*}$ and the set $E^{*}\left(T_{P-P E T}\right)$ of essential P-PET patterns are obtained. Each essential pattern in $E^{*}\left(T_{P-P E T}\right)$ corresponds to a strong diagnostic window of length $n=2^{k}$ as follows: The complete sequence $T_{P-P E T}$ is divided into windows of length $n=2^{k}$. If a window contains an essential pattern, it is called a strong diagnostic window. The windows between two strong windows are joined into one weak diagnostic window. Overall the window structure shown in Figure 2 is obtained, i.e. the strong windows are positioned around the essential patterns, such that the starting index of a window is always a multiple of $2^{k}$.

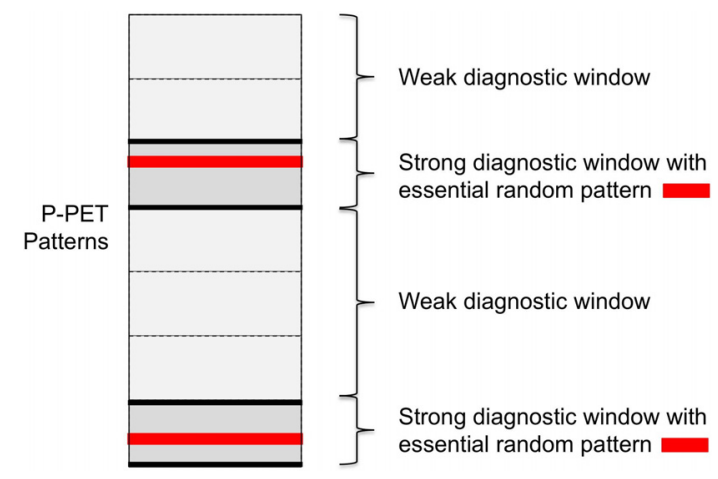

Figure 2. Figure 2: Partitioning a P-PET sequence into diagnostic windows.

Finally, the deterministic patterns $T_{\text {det }}^{*}$ are divided into diagnostic windows of size $2^{k}$, which are also referred to as strong windows.

If faulty signatures appear at the end of both weak and strong windows, then the procedure described in [4][5] is applied to the strong windows only. The patterns in the weak windows are fault simulated to validate the results or to resolve ambiguities. If a faulty signature is observed only at the end of a weak window, then the direct diagnosis procedure described in [7] is applied.

\section{EXPERIMENTAL RESULTS}

The proposed P-PET diagnosis has been evaluated for a set of industrial circuits kindly provided by NXP. The circuit characteristics are listed in Table I. In all experiments reported below, 32-bit MISRs are used, and strong diagnostic windows always contain 32 patterns. 
TABLE I. CIRCUIT CHARACTERISTICS

\begin{tabular}{|c|c|c|c|c|c|}
\hline Circuit & \#Gates & $\begin{array}{c}\text { \#Scan } \\
\text { FFs }\end{array}$ & $\begin{array}{c}\text { \#Scan } \\
\text { Chains }\end{array}$ & $\begin{array}{c}\text { Max. } \\
\text { Length }\end{array}$ & $\begin{array}{c}\text { \# Stuck-at } \\
\text { Faults }\end{array}$ \\
\hline \hline $\mathrm{p} 45 \mathrm{k}$ & 38811 & 2250 & 333 & 97 & 71848 \\
\hline $\mathrm{p} 100 \mathrm{k}$ & 84356 & 5829 & 270 & 53 & 162129 \\
\hline $\mathrm{p} 141 \mathrm{k}$ & 152808 & 10502 & 264 & 45 & 283548 \\
\hline $\mathrm{p} 239 \mathrm{k}$ & 224597 & 18495 & 260 & 61 & 455992 \\
\hline $\mathrm{p} 267 \mathrm{k}$ & 239687 & 16621 & 260 & 62 & 366871 \\
\hline $\mathrm{p} 269 \mathrm{k}$ & 239771 & 16621 & 360 & 62 & 371209 \\
\hline $\mathrm{p} 279 \mathrm{k}$ & 257736 & 17835 & 385 & 59 & 493844 \\
\hline $\mathrm{p} 295 \mathrm{k}$ & 249747 & 18521 & 330 & 62 & 472124 \\
\hline $\mathrm{p} 330 \mathrm{k}$ & 312666 & 18468 & 320 & 64 & 540758 \\
\hline
\end{tabular}

The P-PET approach is compared to a mixed-mode BIST with 4096 and 100000 pseudo-random patterns. Table II shows the achieved fault coverage and compares the cost of deterministic patterns both in terms of the number of patterns and the number of specified bits. The number of specified bits is used as an estimate for the seed memory. As Table II shows, the P-PET scheme greatly reduces the storage requirements for deterministic patterns.

TABLE II. COST OF DETERMINISTIC PATTERNS

\begin{tabular}{|c|c|c|c|c|}
\hline \multirow{2}{*}{ Circuit } & \multirow{2}{*}{$\begin{array}{c}\text { Fault } \\
\end{array}$} & Coverage & \multicolumn{3}{|c|}{ \# Patterns /\# Specified Bits } \\
\cline { 3 - 5 } & & 4096 & 100000 PRP & $\begin{array}{c}\text { P-PET } \\
\text { (b = 24) }\end{array}$ \\
\hline p45k & $99.70 \%$ & $1940 /$ & $303 /$ & $16 /$ \\
& & 52068 & 9064 & 1011 \\
\hline $\mathrm{p} 100 \mathrm{k}$ & $99.56 \%$ & $414 /$ & $118 /$ & $14 /$ \\
& & 60539 & 18843 & 1574 \\
\hline $\mathrm{p} 141 \mathrm{k}$ & $98.85 \%$ & $704 /$ & $494 /$ & $219 /$ \\
& & 375597 & 254410 & 71078 \\
\hline $\mathrm{p} 239 \mathrm{k}$ & $98.84 \%$ & $618 /$ & $431 /$ & $180 /$ \\
& & 162221 & 102537 & 18238 \\
\hline $\mathrm{p} 267 \mathrm{k}$ & $99.60 \%$ & $678 /$ & $578 /$ & $434 /$ \\
& & 393979 & 325800 & 225732 \\
\hline $\mathrm{p} 269 \mathrm{k}$ & $99.58 \%$ & $693 /$ & $533 /$ & $433 /$ \\
& & 396025 & 322150 & 227091 \\
\hline $\mathrm{p} 279 \mathrm{k}$ & $97.89 \%$ & $917 /$ & $668 /$ & $343 /$ \\
& & 397743 & 279820 & 149583 \\
\hline $\mathrm{p} 295 \mathrm{k}$ & $99.15 \%$ & $2553 /$ & $748 /$ & $662 /$ \\
& & 579146 & 362839 & 230737 \\
\hline $\mathrm{p} 330 \mathrm{k}$ & $98.95 \%$ & $5587 /$ & $5191 /$ & $4490 /$ \\
& & 986122 & 866846 & 699460 \\
\hline
\end{tabular}

Table III presents the response data for the P-PET approach as well as the overall memory cost for seed and response data. For comparison Table IV shows the size of the seed memory (SM), the response memory (RM), as well as the overall memory cost $(\Sigma)$ in bytes also for a mixedmode BIST with 4096 and 100000 pseudo-random patterns. For circuits p141k and p330k P-PET has the lowest overall cost. Although the overall cost for P-PET is higher for the other circuits, it can be observed that the reduction in seed memory can compensate the growing response memory to a large extent and keep it in a feasible range. Furthermore, as
TABLE III. TEST AND RESPONSE DATA FOR P-PET $(\ell=8, b=24)$

\begin{tabular}{|c|c|c|c|c|c|}
\hline Circuit & $\begin{array}{c}\text { \# Strong } \\
\text { Windows }\end{array}$ & $\begin{array}{c}\text { \# Weak } \\
\text { Windows }\end{array}$ & $\begin{array}{c}\text { Control } \\
\text { Data } \\
\text { [Byte] }\end{array}$ & $\begin{array}{c}\text { Overall } \\
\text { Response } \\
\text { Data } \\
\text { [Byte] }\end{array}$ & $\begin{array}{c}\text { Seed + } \\
\text { Response } \\
\text { Data } \\
\text { [Byte] }\end{array}$ \\
\hline $\mathrm{p} 45 \mathrm{k}$ & 1844 & 1029 & 4841 & 7714 & 7841 \\
\hline $\mathrm{p} 100 \mathrm{k}$ & 2001 & 1239 & 5253 & 8493 & 8690 \\
\hline $\mathrm{p} 141 \mathrm{k}$ & 3560 & 2847 & 9790 & 16197 & 25082 \\
\hline $\mathrm{p} 239 \mathrm{k}$ & 3928 & 3046 & 10802 & 17776 & 20056 \\
\hline $\mathrm{p} 267 \mathrm{k}$ & 3731 & 2801 & 10261 & 16793 & 45010 \\
\hline $\mathrm{p} 269 \mathrm{k}$ & 3746 & 2795 & 10302 & 16843 & 45230 \\
\hline $\mathrm{p} 279 \mathrm{k}$ & 5179 & 3794 & 14243 & 23216 & 41914 \\
\hline $\mathrm{p} 295 \mathrm{k}$ & 7730 & 5397 & 21258 & 34385 & 63228 \\
\hline $\mathrm{p} 330 \mathrm{k}$ & 3250 & 3949 & 9344 & 16343 & 103776 \\
\hline
\end{tabular}

TABLE IV. COMPARISON OF OVERALL COST [BYTE]

\begin{tabular}{|c|c|c|c|c|c|c|}
\hline \multirow{2}{*}{ Circuit } & \multicolumn{3}{|c|}{$\begin{array}{c}\text { Mixed-Mode BIST with } \\
\text { 4096 PRPP }\end{array}$} & \multicolumn{3}{c|}{$\begin{array}{c}\text { Mixed-Mode BIST with } \\
\text { 100000 PRP }\end{array}$} \\
\cline { 2 - 7 } & SM & RM & $\Sigma$ & SM & RM & $\Sigma$ \\
\hline p45k & 6509 & 189 & 6698 & 1133 & 3680 & 4813 \\
\hline p100k & 7568 & 141 & 7709 & 2356 & 2251 & 4607 \\
\hline p141k & 46950 & 150 & 47100 & 31802 & 2177 & 33979 \\
\hline p239k & 20278 & 148 & 20426 & 12818 & 2320 & 15138 \\
\hline p267k & 49248 & 150 & 49398 & 40725 & 2212 & 42937 \\
\hline p269k & 49504 & 150 & 49654 & 40269 & 2218 & 42487 \\
\hline p279k & 49718 & 157 & 49875 & 34978 & 2760 & 37738 \\
\hline p295k & 72394 & 208 & 72602 & 45355 & 3381 & 48736 \\
\hline p330k & 123266 & 303 & 123569 & 108356 & 2114 & 110470 \\
\hline
\end{tabular}

shown in Table VI, the coverage of non-target faults increases for the P-PET approach.

In order to analyze the diagnostic accuracy, a total of 400 faults have been randomly and uniformly injected into each circuit. The fault set consists of 100 stuck-at faults, 100 crosstalk faults, 100 delay, and 100 wired-AND faults. A fault is considered as correctly diagnosed, if it is one of the top 5 fault candidates in the ranked list after the responses in the fail memory have been analyzed. The depth of the fail memory has been set to 100 . Table V shows the results. The last column shows the improvement due to the additional analysis of weak windows. The same experiment for a mixed-mode BIST with 4096 and 100000 pseudo-random patterns achieved a lower or equal diagnostic resolution for the non-target faults in all cases. Concerning stuck-at faults, the higher number of deterministic stuck-at patterns led to a higher diagnostic resolution for p45k and 4096 pseudorandom patterns.

Finally, Table VI illustrates the contribution of the strong and weak windows to the detection of non-target faults. The column "Strong" reports the number of undetected faults, if only the patterns in the strong windows are evaluated (the mixed-mode BIST with the short pseudo-random sequence is partitioned into strong windows only). The column "Stong+Weak" lists the number of undetected faults, for the complete test. 
TABLE V. Diagnostic RESOLUTION AND IMPACT OF WEAK WINDOWS

\begin{tabular}{|c|c|c|c|c|c|}
\hline Circuit & Stuck-At & $\begin{array}{c}\text { Cross- } \\
\text { talk }\end{array}$ & Delay & $\begin{array}{c}\text { Wired- } \\
\text { And }\end{array}$ & $\begin{array}{c}\text { Improve- } \\
\text { ment by } \\
\text { weak } \\
\text { windows }\end{array}$ \\
\hline \hline $\mathrm{p} 45 \mathrm{k}$ & 99 & 89 & 96 & 91 & 6 \\
\hline $\mathrm{p} 100 \mathrm{k}$ & 98 & 93 & 95 & 100 & 3 \\
\hline $\mathrm{p} 141 \mathrm{k}$ & 99 & 89 & 91 & 95 & 6 \\
\hline $\mathrm{p} 239 \mathrm{k}$ & 98 & 95 & 93 & 100 & 3 \\
\hline $\mathrm{p} 267 \mathrm{k}$ & 99 & 86 & 93 & 95 & 0 \\
\hline $\mathrm{p} 269 \mathrm{k}$ & 98 & 90 & 92 & 99 & 3 \\
\hline $\mathrm{p} 279 \mathrm{k}$ & 95 & 85 & 90 & 94 & 0 \\
\hline $\mathrm{p} 295 \mathrm{k}$ & 95 & 80 & 80 & 86 & 2 \\
\hline $\mathrm{p} 330 \mathrm{k}$ & 95 & 90 & 89 & 94 & 2 \\
\hline
\end{tabular}

TABLE VI. UNDETECTED NON-TARGET FAULTS

\begin{tabular}{|c|c|c|c|c|c|}
\hline \multirow[t]{2}{*}{ Circuit } & \multirow{2}{*}{$\begin{array}{c}4096 \\
\text { PRP } \\
\text { Strong } \\
\end{array}$} & \multicolumn{2}{|c|}{100000 PRP } & \multicolumn{2}{|c|}{ P-PET } \\
\hline & & Strong & $\begin{array}{c}\text { Strong } \\
+ \text { Weak }\end{array}$ & Strong & $\begin{array}{c}\text { Strong + } \\
\text { Weak }\end{array}$ \\
\hline $\mathrm{p} 45 \mathrm{k}$ & 36 & 31 & 27 & 25 & 11 \\
\hline p100k & 17 & 10 & 7 & 10 & 3 \\
\hline $\mathrm{p} 141 \mathrm{k}$ & 26 & 23 & 20 & 15 & 6 \\
\hline p239k & 18 & 11 & 9 & 10 & 6 \\
\hline $\mathrm{p} 267 \mathrm{k}$ & 34 & 23 & 20 & 20 & 8 \\
\hline p269k & 32 & 16 & 16 & 14 & 4 \\
\hline $\mathrm{p} 279 \mathrm{k}$ & 35 & 21 & 21 & 18 & 10 \\
\hline p295k & 52 & 42 & 42 & 38 & 38 \\
\hline p330k & 29 & 26 & 23 & 21 & 13 \\
\hline
\end{tabular}

Comparing the results shows that the weak windows maintain the defect coverage as expected and P-PET has the highest defect coverage.

\section{CONCLUSIONS}

Partial pseudo-exhaustive test offers a high coverage of non-target faults and considerably reduces the storage requirements for deterministic patterns. Partitioning a PPET sequence into strong and weak diagnostic windows supports a fully autonomous BISD combining the high defect coverage with a high diagnostic resolution for nontarget faults as well as feasible storage requirements.

\section{REFERENCES}

[1] M. E. Amyeen et al., "Logic BIST Silicon Debug and Volume Diagnosis Methodology," Proc. IEEE Int. Test Conf. (ITC'11), Anaheim, CA, USA, Sept. 2011, pp. 1-10

[2] Z. Barzilai, D. Coppersmith, A. L. Rosenberg, "Exhaustive Generation of Bit Patterns with Applications to VLSI Self-Testing," IEEE Trans. on Comp., Vol. C-32, No. 2, Feb. 1983, pp. 190- 194.

[3] W.-T. Cheng et al., "Signature based diagnosis for logic BIST," in Proc. IEEE Int. Test Conf. (ITC'06), Santa Clara, CA, USA, 2006, pp. 1-9

[4] A. Cook, M. Elm, H.-J. Wunderlich, U. Abelein, "Structural In-Field Diagnosis for Random Logic Circuits," Proc. Eur. Test Symp. (ETS'11), Trondheim, Norway, May 2011, pp. 111-116

[5] A. Cook, S. Hellebrand, T. Indlekofer, H.-J. Wunderlich, "Diagnostic Test of Robust Circuits," Proc. Asian Test Symp. (ATS'11), New Delhi, India, November 2011.

[6] A. Cook, S. Hellebrand, H.-J. Wunderlich, "Built-in Self-Diagnosis Exploiting Strong Diagnostic Windows in Mixed-Mode Test," Proc. Eur. Test Symp. (ETS'12), Annecy, France, May 2012

[7] S. Holst and H.-J. Wunderlich, "A Diagnosis Algorithm for Extreme Space Compaction," Proc. Design, Automation and Test in Europe (DATE'09), Nice, France, April 20-24, 2009, pp. 1355-1360.

[8] E. J. McCluskey, "Verification Testing - A Pseudoexhaustive Test Technique," IEEE Transactions on Computers, Vol. C-33, No. 6, June 1984 , pp. $541-546$.

[9] A. Mumtaz, M. E. Imhof, H.-J. Wunderlich, "P-PET: Partial PseudoExhaustive Test for High Defect Coverage," Proc. IEEE Int. Test Conf. (ITC'11), Anaheim, CA, USA, Sept. 2011, pp. 1-8 